\title{
Equilíbrio higroscópico de milheto, alpiste e painço: Obtenção e modelagem
}

\author{
Paulo C. Corrêa ${ }^{1}$, Paulo C. A. Júnior ${ }^{2}$, Deise M. Ribeiro ${ }^{3}$ \& Fabrício S. da Silva ${ }^{4}$
}

\begin{abstract}
RESUMO
Para oferecer informações sobre o equilíbrio higroscópico de milheto (Piptatherum miliaceum), alpiste (Phalaris canariensis L.) e painço (Setaria italica), desenvolveu-se este trabalho com o fim de determinar as isotermas de sorção para diferentes níveis de temperatura do ar $\left(20\right.$ e $\left.60{ }^{\circ} \mathrm{C}\right)$ e de atividade de água do produto $(0,20$ a 0,80$)$. A temperatura e a umidade relativa do ar foram controladas por meio de uma unidade condicionadora de ar, modelo Aminco-Aire 150/300 CFM, com fluxo de ar aproximadamente constante de $10 \mathrm{~m}^{3} \mathrm{~min}^{-1} \mathrm{~m}^{-2}$. Os resultados foram avaliados ajustando-se os seguintes modelos matemáticos: Chung-Pfost, Oswin, Smith e Henderson-Modificado. Com base nos resultados obtidos, concluiu-se que a equação proposta por Oswin foi, com seus parâmetros estimados, a que melhor representou os dados experimentais para o milheto, enquanto o modelo de Chung-Pfost o foi para alpiste e painço, quando comparado com os resultados das demais equações avaliadas.
\end{abstract}

Palavras-chave: atividade de água, umidade de equilíbrio, modelos matemáticos

\section{Modeling and obtaining equilibrium moisture content of millet, canary seed and pearl millet}

\begin{abstract}
To provide information on the equilibrium moisture content of millet (Piptatherum miliaceum), canary-seed (Phalaris canariensis L.) and pearl millet (Setaria italica), this study was conducted with the objective of determining the sorption isotherms for different levels of air temperature $\left(20\right.$ and $\left.60{ }^{\circ} \mathrm{C}\right)$ and of water activity of the product $(0.20$ to 0.80$)$. The temperature and the relative humidity of the air (water activity) were controlled through a conditioning unit of air Aminco-Aire 150/300 CFM, with an air flow approximately constant at $10 \mathrm{~m}^{3} \mathrm{~min}^{-1} \mathrm{~m}^{-2}$. The results were evaluated by adjusting to the following mathematical models: Chung-Pfost, Oswin, Smith and Henderson-modified. The results obtained in this work allowed to conclude that the equation proposed by Oswin, with their estimated parameters, was the best to represent the experimental data of millet, while for the experimental data of canary-seed and pearl millet, the ChungPfost model was found to be better, when compared with the results of the other equations.
\end{abstract}

Key words: water activity, equilibrium moisture content, mathematical models 


\section{INTRODUÇÃO}

Em função do alto valor alimentício, o grão do milheto vem despertando enorme interesse para sua utilização em rações para alimentação animal. Atualmente, tem sido muito usado na alimentação de aves, suínos, bovinos e peixes, porém na África e Ásia é bastante empregado na alimentação humana, devido ao acentuado valor protéico.

Além do milheto, outros produtos ricos em proteína, como o alpiste e o painço, com aplicação na alimentação animal, principalmente de pássaros, devem apresentar-se sadios, com elevada qualidade e em perfeito estado de conservação para o consumo.

É imprescindível, para garantir a qualidade e sua conservação, que os grãos sejam transportados e armazenados em locais secos e ventilados e, sobretudo, com baixos teores de umidade; do contrário, o desenvolvimento de microrganismos pode causar fermentações indesejáveis e contaminações por toxinas, que inviabilizam a utilização do produto para consumo humano e animal. Dos microrganismos que colonizam os produtos agrícolas, os fungos são os mais tolerantes a baixas disponibilidades de água e são, conseqüentemente, importantes causas de deterioração (Sauer, 1992).

A disponibilidade de água em materiais biológicos, tais como grãos e frutos, é melhor indicada pela atividade de água (Aw) ou pela umidade de equilíbrio com a temperatura e umidade relativa do ar ambiente. A atividade de água e a umidade relativa, quando atingido o equilíbrio dinâmico, são numericamente iguais (Hall, 1980; Brooker et al., 1992) e sua diminuição com a desidratação do produto contribui para a conservação e uso prolongado do material, uma vez que a retirada de água reduz a sua atividade nos produtos biológicos, retardando o crescimento de microrganismos e impedindo a efetivação de reações bioquímicas que aceleram o processo degenerativo evitando, conseqüentemente, a rápida perda de qualidade do produto.

Christensen \& Kaufmann (1974) estudaram a influência da atividade de água para diversos produtos de origem vegetal, no comportamento dos principais fungos sob condições ótimas de temperatura $\left(26\right.$ a $\left.30^{\circ} \mathrm{C}\right)$ e verificaram que, em geral, atividades de água superiores a 0,70 são favoráveis à sobrevivência e desenvolvimento de fungos do gênero Aspergillus sp; já os fungos do gênero Penicillum sp, carecem de atividades de água maiores necessitando, em sua maioria, de valores superiores a 0,85 .

Os produtos agrícolas possuem a propriedade de realizar trocas de água sob a forma de vapor, com o ambiente que os envolve. Essas trocas podem se dar através do ganho ou da perda de água, fenômenos conhecidos, respectivamente, por adsorção e dessorção, de acordo com as características higroscópicas recíprocas do produto e do ar. A afinidade existente entre a água e os outros componentes (gordura, amido, açúcar, proteínas etc.) de um produto, define sua higroscopicidade (Brooker et al., 1992).

O estudo das curvas de sorção que relacionam a atividade de água e o teor de umidade de equilíbrio a dada temperatura fornece informações fundamentais para adequação das diversas etapas que sucedem a colheita dos produtos agríco- las, por influenciar nos processos de manuseio, secagem, armazenagem e qualidade desses produtos (Afonso Júnior, 2001).

Tendo em vista a importância do conhecimento do comportamento higroscópico de produtos agrícolas para avaliar as variáveis de processamento e definir condições de umidade para manutenção de suas qualidades durante o armazenamento, vários pesquisadores vêm empregando grandes esforços para obtenção de equações e modelos matemáticos que melhor representem este fenômeno (Aguerre et al., 1989; Corrêa et al., 1998; Chen \& Jayas, 1998).

A utilização de equações matemáticas para estimar o teor de umidade de equilíbrio higroscópico apresenta a grande vantagem de predição de valores de atividade de água do produto, em condições ambientais de difícil determinação experimental. A relação entre o teor de umidade de um produto e a umidade relativa de equilíbrio, a dada temperatura, pode ser expressa por meio de curvas características de umidade de equilíbrio, chamadas isotermas. Numerosos são os modelos com capacidade de predizer uma isoterma. Segundo Sun \& Woods (1994), centenas de equações empíricas têm sido desenvolvidas para diversos produtos de origem vegetal. Compilações com modelos matemáticos amplamente utilizados são apresentados por Morey et al. (1995) e Chen \& Jayas (1998).

Chirife \& Iglesias (1978) Mazza \& Jayas (1991) verificaram que, entre os principais modelos matemáticos retratados na literatura para estimar a relação de equilíbrio entre grãos e o ambiente que os envolve, os modelos de ChungPfost, Oswin e Smith foram identificados como mais apropriados para representar as isotermas de adsorção e dessorção de grãos das principais culturas agrícolas; entretanto, diversos autores apontam o modelo proposto por Henderson, modificado por Thompson et al. (1968), como sendo uma equação adequada para descrever o fenômeno (Jayas et al., 1988; Talib et al., 1995).

Ante o exposto, desenvolveu-se este trabalho com o objetivo de analisar quatro modelos matemáticos citados na literatura, a fim de selecionar os mais adequados para predição das isotermas de sorção de umidade de milheto, alpiste e painço, submetidos a diferentes temperaturas e umidades relativas de equilíbrio.

\section{MATERIAL E MÉTODOS}

Este trabalho foi desenvolvido no Laboratório de Propriedades Físicas e Qualidade de Produtos Vegetais do Departamento de Engenharia Agrícola da Universidade Federal de Viçosa, Viçosa, MG.

$\mathrm{O}$ delineamento experimental foi inteiramente casualizado, com diferentes níveis de temperatura $\left(20\right.$ a $\left.60{ }^{\circ} \mathrm{C}\right)$ e de atividade de água $(0,20$ a 0,80$)$ com três repetições.

As condições ambientais para realização dos testes foram obtidas utilizando-se uma unidade condicionadora de atmosfera, modelo Aminco-Aire 150/300 CFM, dotada de dispositivos para o controle da temperatura e umidade relativa do ar fornecido. O equipamento era composto por bandejas removíveis com 


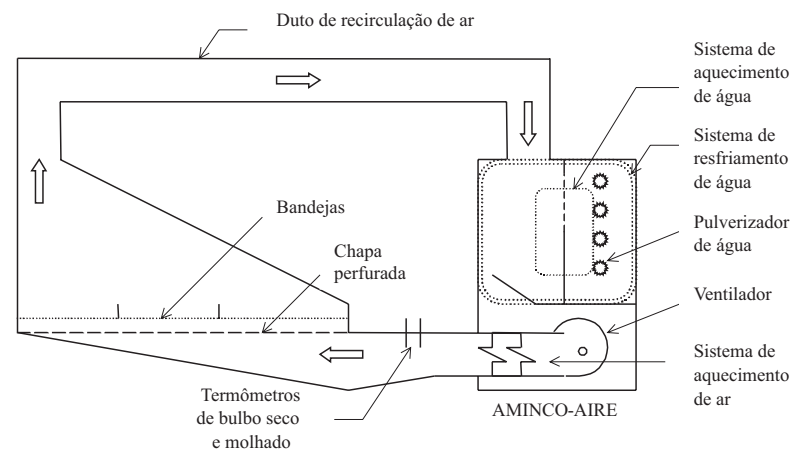

Figura 1. Desenho esquemático do equipamento experimental

fundo telado, para permitir a passagem do ar por entre a massa de produto. O fluxo de ar foi monitorado com o auxílio de um anemômetro de hélice e mantido constante em torno de $10 \mathrm{~m}^{3} \mathrm{~min}^{-1} \mathrm{~m}^{-2}$. A temperatura e a umidade rellativa do ar foram monitoradas por meio de psicrômetro instalado próximo às bandejas contendo as amostras (Figura 1).

As amostras para os testes foram, inicialmente, acondicionadas em sacos de polietileno e armazenadas em câmara fria, a temperatura de $5 \pm 1{ }^{\circ} \mathrm{C}$, e retiradas $12 \mathrm{~h}$ antes do início da operação permitindo, assim, o seu equilíbrio térmico com o ambiente. Para todos os testes, utilizam-se amostras de produto com aproximadamente $200 \mathrm{~g}$, expostas ao fluxo de ar em camada fina, para cada repetição de cada tratamento.

Durante o processo de sorção as bandejas contendo as amostras foram pesadas, periodicamente, visando acompanhar o ganho e a perda de peso durante o processo. Considerou-se que o equilíbrio higroscópico seria alcançado quando a variação da massa das bandejas entre três pesagens fosse igual ou inferior a $0,001 \mathrm{~g}$. Os teores de umidade do produto foram determinados pelo método da estufa, $105 \pm 3{ }^{\circ} \mathrm{C}$, durante 24 h, com três repetições (Brasil, 1992).

Aos dados experimentais foram ajustados quatro modelos matemáticos (Tabela 1), com aplicação reconhecida na predição de isotermas de sorção de umidade.

Para o ajuste dos modelos matemáticos aos dados experimentais de umidade de equilíbrio dos produtos estudados, realizou-se análise de regressão não linear, pelo método Quasi-Newton, utilizando-se o programa computacional Statistica 5.0. Os dados experimentais foram comparados com os valores estimados por cada modelo e produto, pelo erro médio

Tabela 1. Modelos matemáticos empregados na predição das isotermas de sorção de milheto, alpiste e painço

\begin{tabular}{cc}
\hline Chung-Pfost & Modelos \\
\hline Oswin & $\mathrm{Ue}=\mathrm{a}-\mathrm{b} \cdot \ln [-(\mathrm{T}+\mathrm{c}) \cdot \ln (\mathrm{Aw})]$ \\
Smith & $\mathrm{Ue}=[\mathrm{a}+(\mathrm{b} \cdot \mathrm{T})] /[(1-\mathrm{Aw}) / \mathrm{Aw}]^{1 / \mathrm{c}}$ \\
Henderson Modificada & $\mathrm{Ue}=\mathrm{a}-(\mathrm{b} \cdot \mathrm{T})-\mathrm{c} \cdot \ln (1-\mathrm{Aw})$ \\
\hline
\end{tabular}

* Ue: teor de umidade de equilibrio (\% base seca); T: temperatura do ar $\left({ }^{\circ} \mathrm{C}\right)$; Aw: atividade de água (decimal); a, b e c: constantes que dependem da natureza do produto relativo (P) e erro médio estimado (SE), conforme descrito a seguir:

$$
\begin{aligned}
& \mathrm{P}=\frac{1}{\mathrm{n}} \cdot \sum \frac{|\mathrm{Y}-\hat{\mathrm{Y}}|}{\mathrm{Y}} \\
& \mathrm{SE}=\sqrt{\frac{\sum(\mathrm{Y}-\hat{\mathrm{Y}})^{2}}{\mathrm{GLR}}}
\end{aligned}
$$

em que

$$
\begin{array}{ll}
\mathrm{n} & \text { - numero de observações } \\
\mathrm{Y} & \text { - valor experimental } \\
\hat{\mathrm{Y}} & \text { - valor calculado } \\
\text { GLR } & \text { - grau de liberdade }
\end{array}
$$

O grau de ajuste dos modelos matemáticos aos dados experimentais, baseou-se na magnitude do coeficiente de determinação ajustado (variância explicada), na magnitude dos erros médios relativos e estimados e na análise do comportamento da distribuição dos resíduos.

\section{RESULTADOS E DISCUSSÃO}

\begin{tabular}{|c|c|c|c|c|}
\hline \multirow[b]{2}{*}{ Parâmetros } & \multicolumn{4}{|c|}{ Modelos Matemáticos } \\
\hline & Chung-Pfost & Oswin & Smith & $\begin{array}{l}\text { Henderson } \\
\text { Modificada }\end{array}$ \\
\hline \multicolumn{5}{|c|}{ Milheto } \\
\hline $\mathrm{a}$ & 43,2275 & 21,1184 & 12,8482 & $3,8727 \times 10^{-4}$ \\
\hline $\mathrm{b}$ & 8,8577 & $-0,2005$ & 0,2227 & 7,1834 \\
\hline c & 1,3791 & 2,0475 & 13,2314 & 1,4161 \\
\hline $\mathrm{R}^{2}$ & 0,9400 & 0,9770 & 0,9651 & 0,9587 \\
\hline $\mathrm{P}$ & 0,1180 & 0,0688 & 0,0912 & 0,0958 \\
\hline SE & 4,4467 & 2,7558 & 3,3932 & 3,6920 \\
\hline $\begin{array}{l}\text { Distribuição } \\
\text { do Resíduo }\end{array}$ & tendencioso & aleatório & tendencioso & aleatório \\
\hline \multicolumn{5}{|c|}{ Alpiste } \\
\hline A & 43,0279 & 13,9987 & 8,6322 & $2,2588 \times 10^{-4}$ \\
\hline B & 5,5447 & $-0,0160$ & 0,0207 & 46,1543 \\
\hline C & 259,6436 & 3,4872 & 7,7062 & 1,4379 \\
\hline $\mathrm{R}^{2}$ & 0,9982 & 0,9942 & 0,9818 & 0,5816 \\
\hline$P$ & 0,0091 & 0,0166 & 0,0290 & 0,1741 \\
\hline SE & 0,4524 & 0,8154 & 1,4430 & 8,6885 \\
\hline $\begin{array}{l}\text { Distribuição } \\
\text { do Resíduo }\end{array}$ & aleatório & tendencioso & tendencioso & tendencioso \\
\hline \multicolumn{5}{|c|}{ Painço } \\
\hline a & 47,5696 & 14,7753 & 8,6760 & $9,1851 \times 10^{-5}$ \\
\hline$b$ & 6,2570 & $-0,0192$ & 0,0230 & 16,1669 \\
\hline c & 262,3067 & 3,2652 & 8,7098 & 1,9275 \\
\hline $\mathrm{R}^{2}$ & 0,9987 & 0,9946 & 0,9846 & 0,7429 \\
\hline$P$ & 0,0084 & 0,0173 & 0,0288 & 0,1185 \\
\hline SE & 0,4322 & 0,8867 & 1,4947 & 6,1078 \\
\hline $\begin{array}{l}\text { Distribuição } \\
\text { do Resíduo }\end{array}$ & aleatório & tendencioso & tendencioso & tendencioso \\
\hline
\end{tabular}

Na Tabela 2 tem-se o resumo dos modelos ajustados por meio de regressão não linear, aos dados experimentais de

Tabela 2. Parâmetros das equações ajustadas para se calcular os teores de umidade de equilíbrio higroscópico de milheto, alpiste e painço, em função da temperatura do ar (T) e da atividade de água do produto (Aw) 

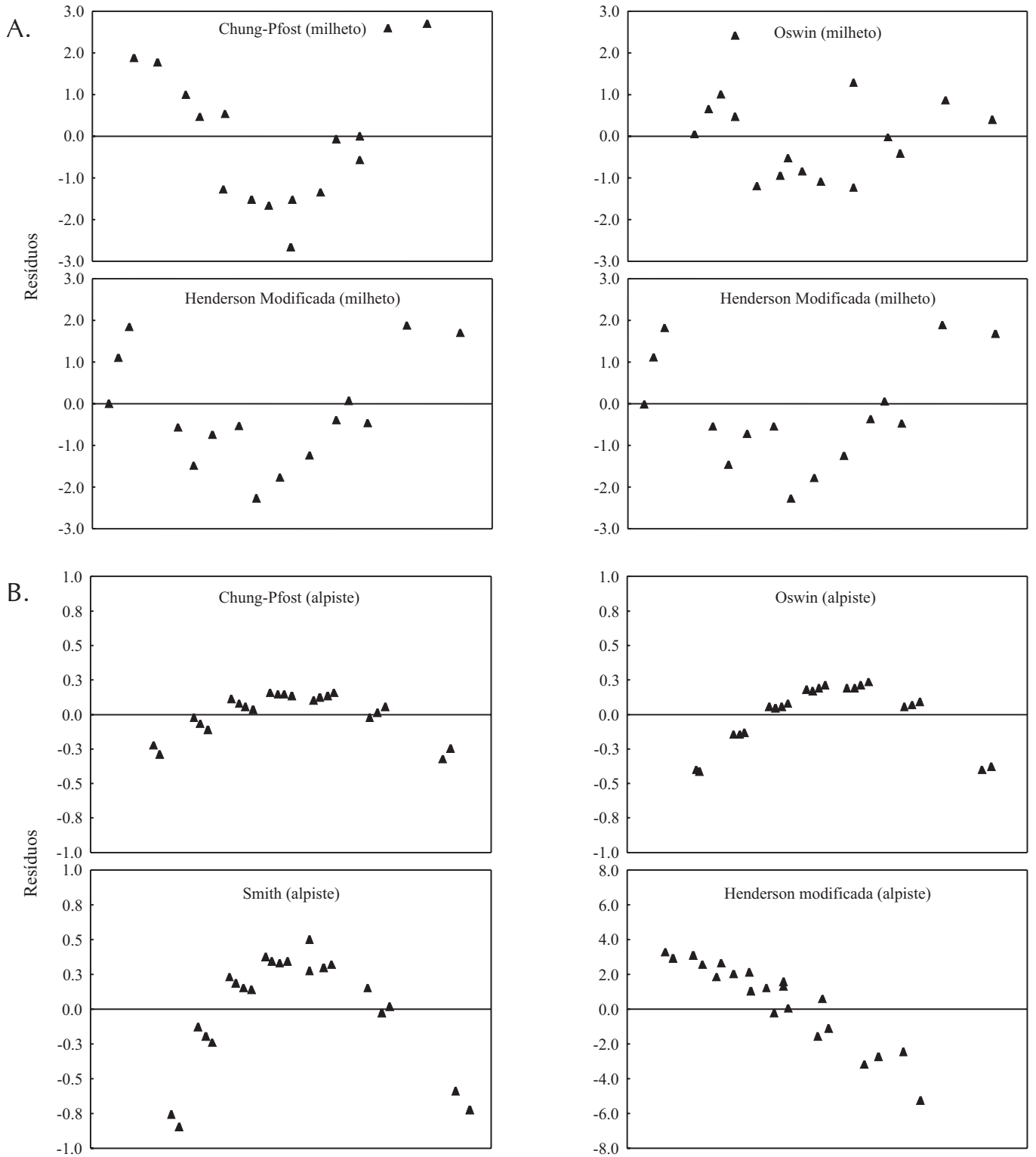

C.
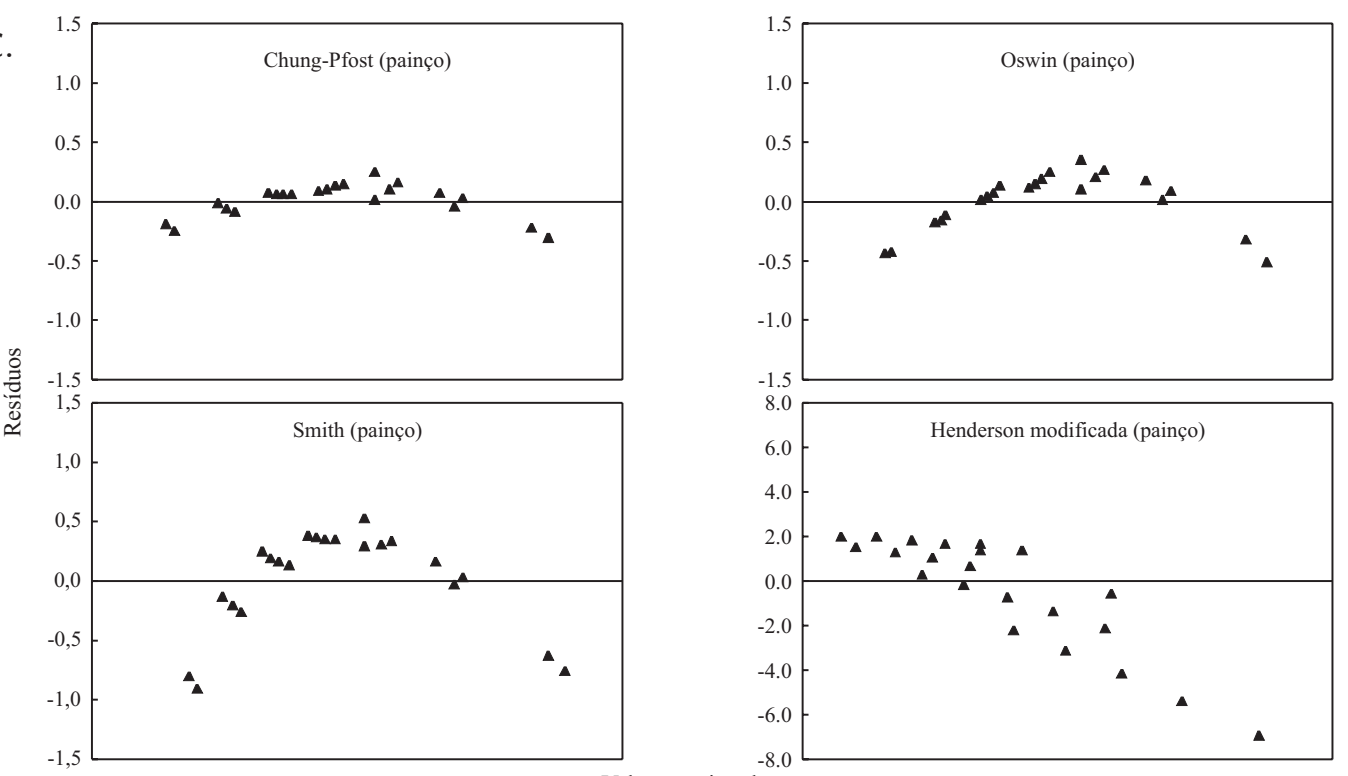

Figura 2. Distribuição dos resíduos de cada modelo estudado para milheto (A), alpiste (B) e painço (C) em função dos valores estimados

R. Bras. Eng. Agríc. Ambiental, v.10, n.1, p.162-167, 2006. 
equilíbrio higroscópico de milheto, alpiste e painço.

Observa-se que a equação com base no modelo de Oswin foi a que melhor se ajustou aos dados experimentais de equilíbrio higroscópico de milheto, com coeficiente de determinação ajustado de 0,9770, erro médio relativo de 0,0687 e erro médio estimado de 2,7556, apresentando melhor comportamento para representar as isotermas de sorção de milheto; entretanto, a análise dos resultados apresentados na Tabela 2, indica que o modelo de Chung-Pfost se mostrou adequado para fornecer estimativas satisfatórias para o teor de umidade de equilíbrio higroscópico dos grãos de alpiste e painço, por seus reduzidos valores para os erros médios relativos e estimados e, ainda, elevados coeficientes de determinação ajustados, quando comparados com os resultados obtidos para os demais modelos testados.

Os resíduos, baseados na diferença entre os valores experimentais e os estimados para cada modelo matemático ajustado, são apresentados na Figura 2.

Constata-se que os valores residuais para o modelo de Oswin (Figura 2A) apresentaram comportamento aleatório, indicando ser este modelo mais indicado para descrição da relação de higroscopicidade do milheto e o ambiente que o envolve. No entanto, para os modelos de Chung-Pfost, Smith e Henderson-Modificada, observa-se uma distribuição tendenciosa dos resíduos, sugerindo que essas equações representam, de forma menos satisfatória, o fenômeno estudado. Nas Figuras 2B e 2C vê-se, para os grãos de alpiste e painço, que o modelo de Chung-Pfost foi o único a apresentar comportamento aleatório dos valores residuais, em função dos teores de umidade de equilíbrio higroscópico estimados.

Embora várias teorias tenham sido propostas para predizer o comportamento higroscópico de produtos biológicos, na maioria das vezes as relações matemáticas empíricas se têm apresentado como melhores opções para predizer o fenômeno, vez que nenhum modelo teórico desenvolvido foi capaz, até o momento, de predizer com precisão o teor de umidade de equilíbrio desses produtos para todas as faixas de temperatura e atividade de água (Brooker et al., 1992).

$\mathrm{Na}$ Figura 3 apresentam-se gráficos em que se comparam os valores de teores de umidade de equilíbrio experimentais e os estimados, pelos modelos que melhor representaram o comportamento higroscópico dos produtos avaliados em função da atividade de água do material e da temperatura do ar. Verificam-se, na Figura 3A, não apenas o ajuste adequado da equação de Oswin mas, também, a pequena tendência de dispersão dos valores estimados em relação aos dados experimentais de milheto. Da mesma forma observa-se, nas Figuras 3B e 3C, que o modelo de Chung-Pfost apresentou pequeno desvio entre os valores experimentais e os estimados de grãos de alpiste e painço, respectivamente, representando, de maneira satisfatória, o processo de equilíbrio higroscópico desses produtos. Estes resultados concordam com Chirife \& Iglesias (1978) e Mazza \& Jayas (1991), os quais verificaram que os modelos matemáticos de Chung-Pfost, Oswin e Smith são os mais apropriados para representar as isotermas de adsorção e dessorção de grãos das principais culturas agrícolas.
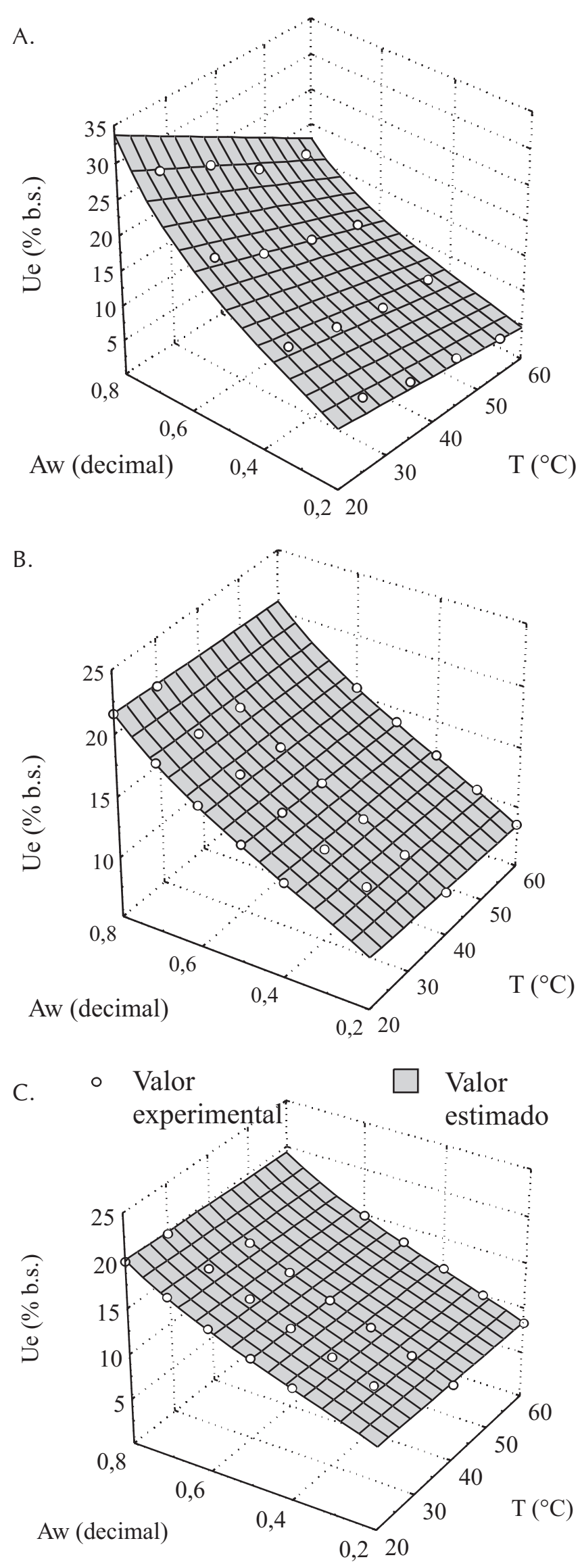

Figura 3. Valores experimentais e estimados do teor de umidade de equilíbrio para grãos de milheto, alpiste e painço, utilizando-se os modelos de Oswin (A) e de Chung-Pfost (B e C), respectivamente 


\section{CONCLUSÕES}

1. A equação proposta por Oswin foi a que melhor representou os dados experimentais de milheto, quando comparada com as equações de Chung-Pfost, Smith e HendersonModificada.

2. A equação proposta por Chung-Pfost foi a que melhor representou os dados experimentais de alpiste e painço, quando comparada com as equações de Oswin, Smith e Henderson-Modificada.

\section{LITERATURA CITADA}

Afonso Júnior, P. C. Influência do método de preparo e condições de secagem sobre a qualidade do café durante o período de armazenagem. Viçosa: UFV, 2001. 351p. Tese Doutorado

Aguerre, R. J.; Suárez, C.; Viollaz P. E. Modeling temperature dependence of food sorption isotherms. Lebensmittel-Wissenschaft und-Technologie, London, v.22, n.1, p.1-5, 1989.

Brasil. Ministério da Agricultura e Reforma Agrária. Regras para análise de sementes. Brasília: DNDV/CLAV, 1992. 365p.

Brooker, D. B.; Bakker-Arkena, F. W.; Hall, C. W. Drying and storage of grains and oilseeds. New York: AVI, 1992. 450p.

Chen, C.; Jayas, D. S. Evaluation of the GAB equation for the isotherms of agricultural products. Transactions of ASAE, St. Joseph, v.41, n.6, p.1755-1760, 1998.

Chirife, J.; Iglesias, H. A. Equations for fitting water sorption isotherms of foods: Part 1 - a review. Journal of Food Technology, London, v.13, n.2, p.159-174, 1978.
Christensen, C. M.; Kaufmann, H. H. Microflora. In: Christensen, C. M. Storage of cereal grain and their products. St. Paul: American Association of Cereal Chemists, 1974. p.158-192.

Corrêa, P. C.; Martins, J. H.; Christ, D.; Mantovani, B. H. M. Curvas de dessorção e calor latente de vaporização para as sementes de milho pipoca (Zea mays). Revista Brasileira de Engenharia Agrícola e Ambiental, Campina Grande, v.2, n.1, p.75-79, 1998.

Hall, C. W. Drying and storage of agricultural crops. Westport: The AVI Publishing Company, 1980. 381p.

Jayas, D. S.; Kukelko, D. A.; White, N. D. G. Equilibrium moisture-equilibrium relative humidity relationship for canola meal. Transactions of ASAE, St. Joseph, v.31, n.5, p.1585-1593, 1988.

Mazza, G.; Jayas, D. S. Equilibrium moisture characteristics of sunflower seeds, hulls, and kernels. Transactions of ASAE, St. Joseph, v.34, n.2, p.532-538, 1991.

Morey, V.; Wilcke, W. F.; Meronuck, R. A.; Lang, J. P. Relationship between equilibrium relative humidity and deterioration of shelled corn. Transactions of ASAE, St. Joseph, v.38, n.4, p.1139-1145, 1995.

Sauer, D. B. Storage of cereal grains and their products. St Paul: American Association of Cereal Chemists, 1992. 615p.

Sun, D. W.; Woods, J. L. The selection of sorption isotherm equations for wheat based on the fitting of available data. Journal of Stored Product Research, London, v.30, n.1, p.27-43, 1994.

Talib, M. Z. M.; Daud, W. R. W.; Ibrahim, M. H. Moisture desorption isotherms of cocoa beans. Transactions of ASAE, St. Joseph, v.38, n.4, p.1153-1155, 1995.

Thompson, T. L.; Peart, R. M.; Foster, G. H. Mathematical simulation of corn drying - a new model. Transactions of ASAE, St. Joseph, v.11, n.4, p.582-586, 1968. 